\title{
Analysis of Chirped Oscillators Under Injection Signals
}

\author{
Franco Ramírez, Sergio Sancho, Mabel Pontón, Almudena Suárez \\ Dept. of Communications Engineering, University of Cantabria, Santander, Spain
}

\begin{abstract}
An in-depth investigation of the response of chirped oscillators under injection signals is presented. The study confirms the oscillator capability to detect the input-signal frequencies, demonstrated in former works. To overcome previous analysis limitations, a new formulation is presented, which is able to accurately predict the system dynamics in both locked and unlocked conditions. It describes the chirped oscillator in the envelope domain, where two time scales are used, one associated with the oscillator control voltage and the other associated with the beat frequency. Under sufficient input amplitude, a dynamic synchronization interval is generated about the input-signal frequency. In this interval, the circuit operates at the input frequency, with a phase shift that varies slowly at the rate of the control voltage. The formulation demonstrates the possibility of detecting the input-signal frequency from the dynamics of the beat frequency, which increases the system sensitivity. All the results have been validated with both full circuit-level simulations and measurements.
\end{abstract}

Index Terms - Chirp signal, injection locking, beat frequency

\section{INTRODUCTION}

The interesting works [1]-[2] successfully investigated the possible application of chirped oscillators under the injection of wireless signals in cognitive radio, for spectrum sensing [3]-[4] and frequency demodulation. This conclusion relies on the capability of the chirped oscillator to get locked to an input signal having a frequency comprised within its dynamic oscillation-frequency interval, which is actually achieved with a certain input-signal power. The theoretical analysis in [2] predicts a linear variation of the chirp-oscillator frequency within the synchronization interval, though this frequency should agree with that of the input source. This analysis is performed in terms of the oscillation frequency, instead of the chirp-signal voltage, that constitutes the actual control variable.

Here an in-depth investigation of the dynamics of the chirpsignal oscillator under wireless injection is presented. It is based on an envelope-domain, semi-analytical formulation of the injected circuit, using an oscillator model extracted from harmonic balance (HB) [5]-[6]. The variations of the dynamic oscillation under both locked and unlocked conditions are analyzed using two time scales, one associated with the control voltage of the chirped oscillator, and the other associated with the beat frequency. This avoids the computational difficulties of a full envelope-domain formulation [7]-[9], which must cover the slow sawtooth-signal period (on the order of $\mathrm{ms}$ ) at a small integration time step, accounting for the beat frequency outside the synchronization time interval. By means of the envelope-domain formulation with two time scales, it is demonstrated that the chirp oscillator is able to detect a wireless signal even when its power is below the one required to reach a locked state during the chirp-signal period. The possible system application as a receiver of frequency-modulated signals with carriers within the chirped-oscillator frequency band is investigated, which may, for instance, have interest in sensor networks.

\section{FORMULATION OF THE INJECTED CHIRPED OSCILLATOR}

Consider the oscillator in Fig. 1, which exhibits the freerunning oscillation frequency $\omega_{o o}$ and amplitude $V_{o}$ at the control voltage $\eta_{0}$. A sawtooth waveform $\eta(t)$ will be introduced at the the control node and one or more input signals will be injected, modeled with their equivalent currents $i_{g, k}$, where $k=1$ to $N$. The whole system will be modeled with an envelope-domain outer-tier equation at the fundamental frequency, considering the higher harmonic terms in an inner tier [6]. Initially, a single input signal $i_{g}$ will be assumed. The first harmonic of the output voltage is expressed as:

$$
v(t) \approx\left[V_{o}+\Delta V(t)\right] e^{j \phi(t)} e^{j \omega_{i} t}
$$

where $\omega_{i}$ is the input frequency. The outer-tier equation, in terms of the admittance function $Y$ at $\omega_{i}$, at the output node, is:

$$
Y\left(V_{o}+\Delta V, \omega_{i}+s / j, \eta_{0}+\Delta \eta, I_{g}^{r}, I_{g}^{i}\right)\left(V_{o}+\Delta V\right) e^{j \phi}=0
$$

where the superscripts indicate real and imaginary parts, $s$ is the time-derivative operator, $\eta_{0}$ is the middle point of the chirp-voltage excursion, $\eta_{0}=\left(\eta_{\min }+\eta_{\max }\right) / 2$ and $\Delta \eta$ is the time-varying increment with respect to $\eta_{0}$. Assuming a quasilinear oscillator response versus $\eta$ (as expected in the chirped oscillator) and low input amplitude $\left|I_{g}\right|$, the oscillator can be linearized about $\eta_{0}, V_{o}$ and $\omega_{o o}$. Neglecting the time-varying term $\Delta \dot{V}$ one obtains:

$$
\begin{aligned}
& \dot{\varphi}=\omega_{o}(\Delta \eta)-\omega_{i}+K_{s} \sin \varphi+K_{c} \cos \varphi, \\
& \varphi \equiv \phi-\phi_{i}, \quad \omega_{o}(\Delta \eta) \equiv \omega_{o o}+K_{V} \Delta \eta
\end{aligned}
$$

where $\phi_{i}$ is the input-signal phase and the coefficients $K_{s}, K_{c}$ and $K_{V}$ correspond to those given in [10]-[11]. In each cycle of the chirp signal, the tuning voltage grows linearly as $\Delta \eta=$ $p t$. Taking into account the slow oscillation-frequency modulation due to $\Delta \eta$ and the possible existence of a beat frequency $\omega_{b}$, the nonlinear equation (3) can be solved, expressing the phase variable $\varphi(t)$ in a Fourier series with two time scales:

$$
\begin{aligned}
& \varphi(t)=\hat{\varphi}(t, t), \\
& \hat{\varphi}\left(t_{1}, t_{2}\right)=\omega_{b}\left(t_{1}\right) t_{2}+\sum_{k} P_{k}\left(t_{1}\right) e^{j k \omega_{b}\left(t_{1}\right) t_{2}}
\end{aligned}
$$


The slow scale $t_{1}$, associated with the time-varying tuning voltage $\Delta \eta(t)$, modulates the components of expression (4). The faster scale $t_{2}$ is associated with the beat frequency $\omega_{b}$, which varies with $t_{1}$. Introducing (4) in (1), the instantaneous frequency shift $\dot{\varphi}(t)$ can be determined:

$$
\begin{aligned}
\dot{\varphi}(t) & =\frac{\partial \hat{\varphi}(t, t)}{\partial t_{1}}+\frac{\partial \hat{\varphi}(t, t)}{\partial t_{2}}=\frac{\partial \hat{\varphi}(t, t)}{\partial t_{1}}+ \\
& +\omega_{b}\left(t_{1}\right)+\sum_{k} j k \omega_{b}\left(t_{1}\right) P_{k}\left(t_{1}\right) e^{j k \omega_{b}\left(t_{1}\right) t}, \quad t_{1}=t
\end{aligned}
$$

In the time intervals with unlocked behavior, the partial derivative with respect to $t_{1}$ can be neglected since $\omega_{b}\left(t_{1}\right)$ and $P_{k}\left(t_{1}\right)$ are slowly-varying components. Then, equation (5) predicts that at each $t_{1}, \dot{\varphi}(t)$ is a periodic signal centered at $\omega_{b}\left(t_{1}\right)$ with period $T_{b}\left(t_{1}\right)=2 \pi / \omega_{b}\left(t_{1}\right)$.

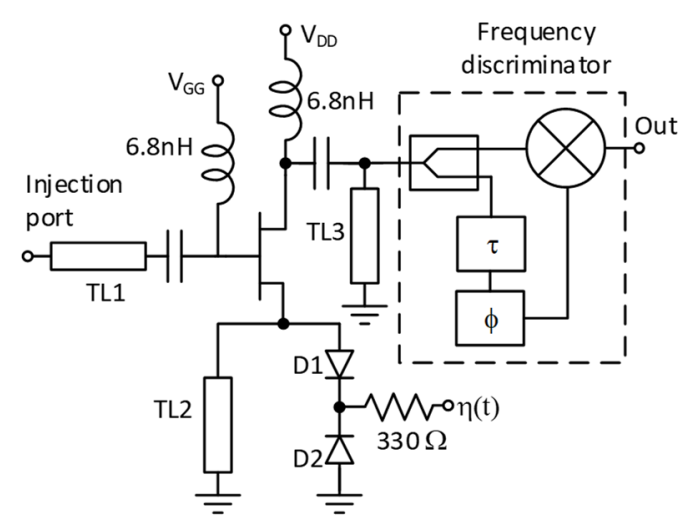

(a)

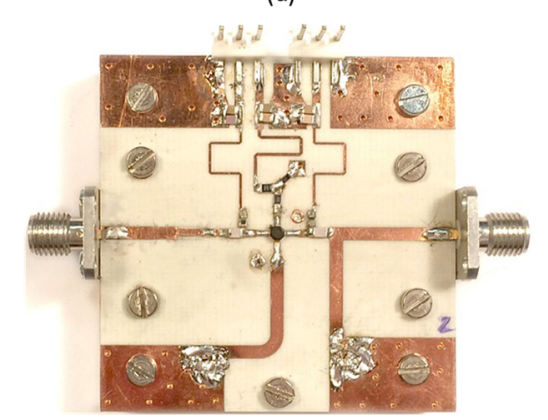

(b)

Fig. 1. Chirped oscillator based on the transistor NE3210S01. It is controlled by the tuning voltage $\eta(t)$, corresponding to a sawtooth waveform. (a) Schematic of the oscillator circuit. (b) Photograph.

Now, operation under locked conditions will be assumed. In this case, $\omega_{b}\left(t_{1}\right)=0$ and $\hat{\varphi}\left(t_{1}, t_{2}\right)=P_{0}\left(t_{1}\right)$. For each $t_{1}$, equations (3) and (4) predict a single-tone spectrum at $\omega_{i}$, and the instantaneous frequency shift becomes:

$$
\dot{\varphi}(t)=\frac{\partial \hat{\varphi}(t, t)}{\partial t_{1}}+\frac{\partial \hat{\varphi}(t, t)}{\partial t_{2}}=\frac{d P_{0}(t)}{d t_{1}} \equiv \dot{P}_{0}(t)
$$

where $\dot{P}_{0}(t)$ is a very small frequency, just due to the slow variation of the tuning voltage $\Delta \eta(t)$.
The analysis method has been initially validated through comparison with circuit-level simulations at a chirp frequency of $10 \mathrm{kHz}$. This is the lowest value that could be considered in the circuit-level analysis, due to the huge computational cost of a long simulation time interval under relatively high $\omega_{b}$. The input power is $P_{\text {in }}=-8 \mathrm{dBm}$. Fig. 2 shows the dynamic variation of the output amplitude shift $\Delta V$ during the period of the chirp signal for $f_{i}=5.45 \mathrm{GHz}$, obtained through circuitlevel simulations in (a), and with the formulation (3)-(6) in (b). In the synchronization interval the output amplitude approaches the ellipsoidal curve that would be obtained in static conditions, that is, when varying $\eta$ in the interval $\left(\eta_{\text {min }}, \eta_{\text {max }}\right)$. This static curve has also been represented as a reference.

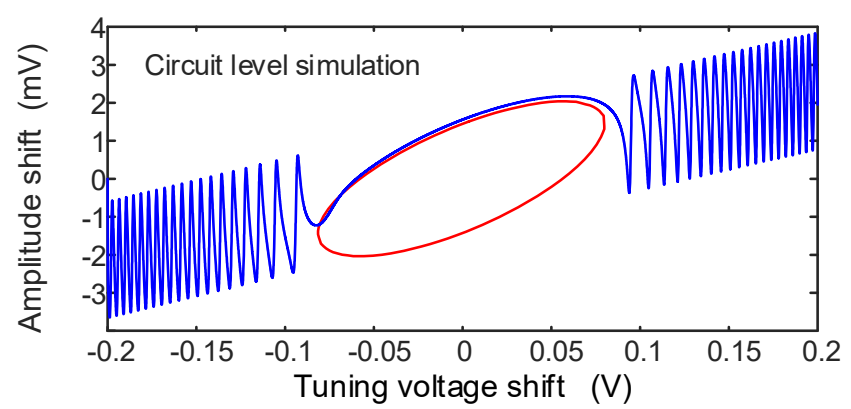

(a)

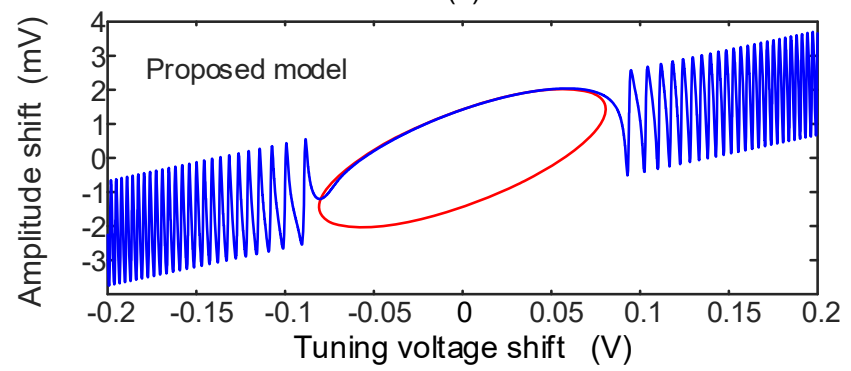

(b)

Fig. 2 Dynamic variation of the oscillation magnitude at the chirp frequency $10 \mathrm{kHz}$. (a) Circuit-level simulations. (b) Equations (3)-(6).

Within the synchronization interval, $\varphi\left[\eta_{0}+\Delta \eta(t)\right]$ approaches the phase relationship $\varphi(\eta)$ that would be obtained in static conditions, so the oscillation frequency only exhibits a slow time variation, as predicted by (6). The synchronization interval is always centered about the tuning voltage $\eta_{c}$ that would provide an oscillation at the input frequency $\omega_{i}$ in static conditions. Fig. 3 shows the dynamic frequency variation obtained through circuit-level simulations and with (3)-(6). In the unlocked time intervals, the instantaneous frequency is given by (5). The magnitude of $\omega_{b}\left(t_{1}\right)$ decreases as $\omega_{o}(\Delta \eta)$ shifts towards the synchronization band. In the unlocked intervals, the average frequency increases with time due to the positive slope of $\Delta \eta=p t$.

In the presence of $N$ input carriers $i_{g, k}$, injection-locking intervals may still be detected. Equation (2) can be extended to this situation in the form: 


$$
\begin{gathered}
Y_{\eta} \Delta \eta+Y_{V} \Delta V+Y_{\omega}\left(\Delta \omega_{0}+\dot{\phi}-j \frac{\Delta \dot{V}}{V_{o}+\Delta V}\right)+\sum_{k=1}^{N} Y_{g k}=0 \\
Y_{g k}=I_{k}\left(\frac{\partial Y}{\partial I_{k, 1}} e^{j \varphi_{k}}+\frac{\partial Y}{\partial I_{k,-1}} e^{-j \varphi_{k}}\right), I_{k, \pm 1}=I_{k} e^{ \pm j \phi_{i k}} \\
\varphi_{k}=\phi_{i k}-\phi-\Delta \omega_{k} t, \quad \Delta \omega_{k}=\omega_{k}-\omega_{1}
\end{gathered}
$$

where $I_{k}, \phi_{i k}$ and $\omega_{k}$ are the amplitude, phase and frequency of each carrier. With two (or more) input carriers, the instantaneous frequency will vary in a nearly periodic (or quasiperiodic) manner within the synchronization intervals.

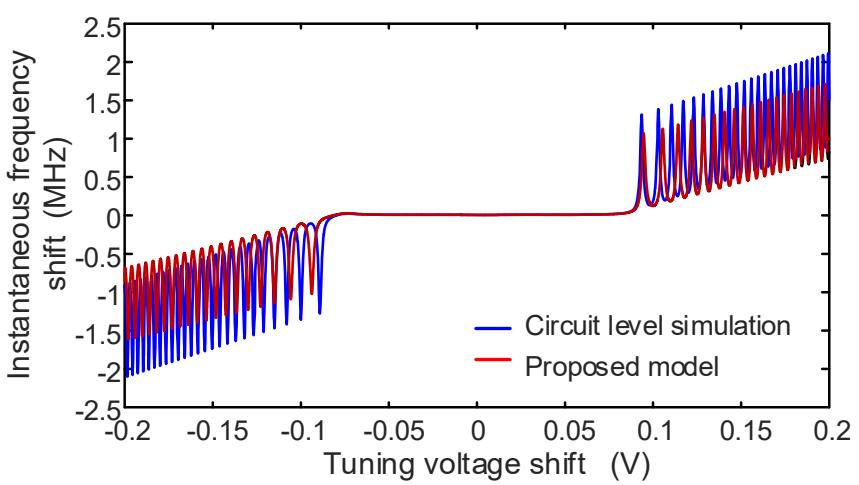

Fig. 3. Dynamic variation of the oscillation frequency at the chirp frequency $10 \mathrm{kHz}$. Comparison between equations (3)-(6) and circuit-level simulation.

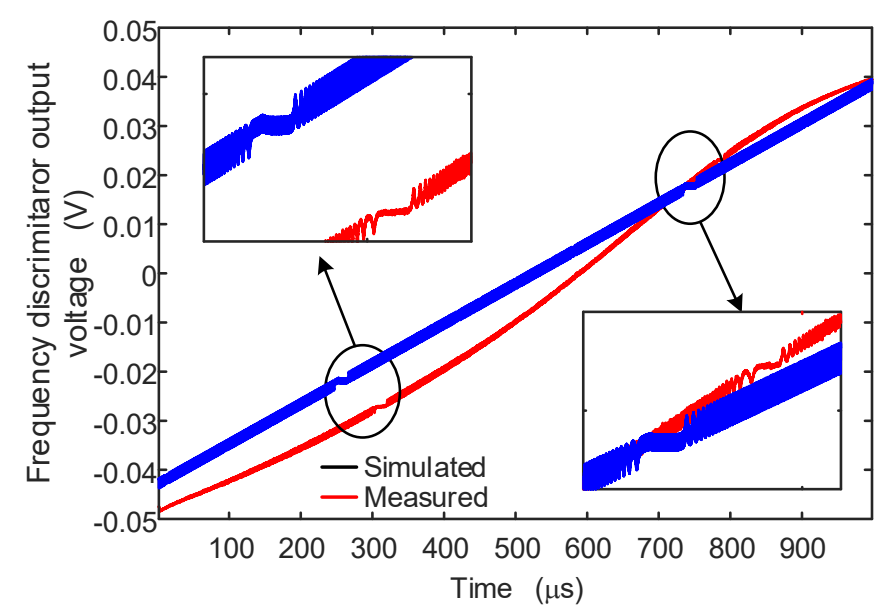

Fig. 4 Comparison of (7) with experimental measurements, under a chirp signal of $1 \mathrm{kHz}$, in the presence of two input signals at $f_{1}=5.461 \mathrm{GHz}$ and $f_{2}=5.479 \mathrm{GHz}$

At the low chirp frequency $1 \mathrm{kHz}$, no circuit-level envelope-domain simulations were possible. Fig. 4 compares the results of (7) with experimental measurements under a chirp signal of $1 \mathrm{kHz}$, in the presence of two inputs at $f_{1}=5.461$ $\mathrm{GHz}$ and $f_{2}=5.479 \mathrm{GHz}$. The vertical axis corresponds to the voltage signal obtained at the output of the frequency discriminator in Fig. 1. During the chirp signal period, the circuit gets locked to the first frequency and then two the second frequency. The time shift between the results of the new analysis method and the measurements is attributed to inaccuracies in the models of the circuit elements, and, as a result, in the derivatives of the admittance function. Note that the new method exhibits an excellent agreement with circuit-level simulations, as shown in Fig. 2 and Fig. 3.

In the case of a single frequency-modulated input signal, it is possible to demodulate this signal during the synchronization time interval. Fig. 5 compares analysis and measurement results after passing the oscillator output through a frequency discriminator and a high-pass filter [2]. The detection of fast modulations requires a low chirp frequency. The detection method can be applied to both analog and digital frequency modulated signals.

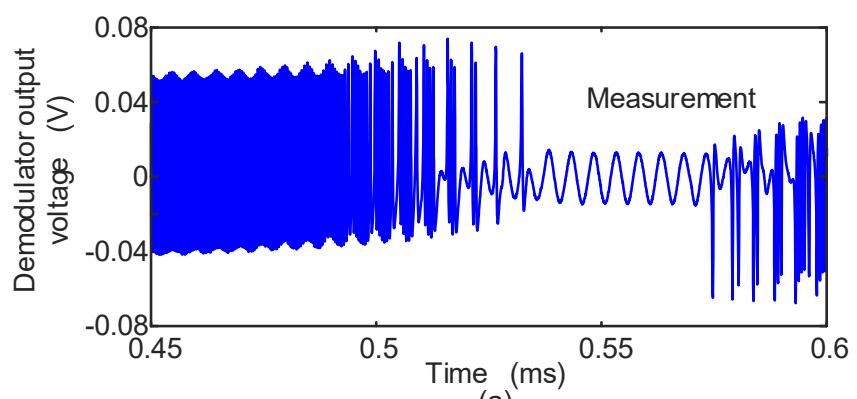

(a)

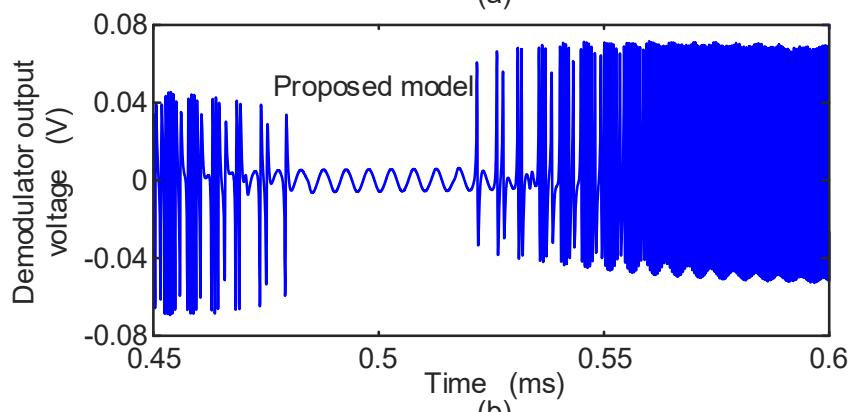

(b)

Fig. 5 Demodulation of an input FM signal during the locking time interval. $\left(f_{\text {mod }}=200 \mathrm{kHz}, \Delta f=500 \mathrm{kHz}\right)$

\section{OPERATION UNDER UNLOCKED CONDITIONS}

The two boundaries of the synchronization interval correspond to the time values at which the beat frequency decreases to zero and grows from zero, respectively. The mathematical condition for this dynamic bifurcation can be expressed as $|\dot{\varphi}(t)|=\varepsilon$, where $\varepsilon$ is a threshold used for the numerical detection. This threshold must fulfill $\left|\dot{P}_{0}(t)\right|<\varepsilon, \forall t$. When progressively reducing the input current, a value should be reached $I_{g \min }$ at which the two time points merge in a single one $t_{c}$, which fulfills $\eta_{0}+\Delta \eta\left(t_{c}\right) \approx \eta\left(\omega_{i}\right)$, where $\eta\left(\omega_{i}\right)$ is the tuning-voltage value that would provide $\omega_{i}$ in static conditions. With input current below $I_{g m i n}$, detecting an input signal is still possible, taking into account the impact of this signal on the sign of the beat frequency. In this case, the frequency pulling can be neglected due to the small amplitude of $I_{g}$, so the beat frequency can be expressed as:

$$
\omega_{b}\left(t_{1}\right) \approx \omega_{o}(\Delta \eta)-\omega_{i}=K_{V} p t_{1}-\omega_{i}
$$


Then applying equation (5), the second derivative of the instantaneous phase is given by:

$\ddot{\varphi}(t) \approx K_{V} p+\sum_{k}-k^{2} \omega_{b}\left(t_{1}\right)^{2} P_{k}\left(t_{1}\right) e^{j k \omega_{b}\left(t_{1}\right) t}, t_{1}=t$

where the partial derivative in the slow scale $t_{1}$ appearing in (5) has been neglected. The beat frequency $\omega_{b}\left(t_{1}\right)$ changes sign at the time $t_{c}$ for which $\omega_{o}=\omega_{i}$. Thus, $\ddot{\varphi}(t)$ necessarily slows down near $t_{c}$, becoming nearly constant at $\ddot{\varphi}\left(t_{c}\right) \approx$ $K_{V} p$. In the presence of several input signals, the oscillator instantaneous frequency will also exhibit slower variations in the neighborhood of each input frequency. Figs. 6(a)-(b) show the simulated and measured signal, after passing through a frequency discriminator and a high-pass filter, under $P_{\text {in }}=$ $-50 \mathrm{dBm}$ at $f_{1}=5.461 \mathrm{GHz}$ and $f_{2}=5.479 \mathrm{GHz}$. Fig. 6(b) shows the result of passing this demodulated signal through an envelope detector. Note that the input signal is directly introduced into the oscillator, without any previous amplification stage.

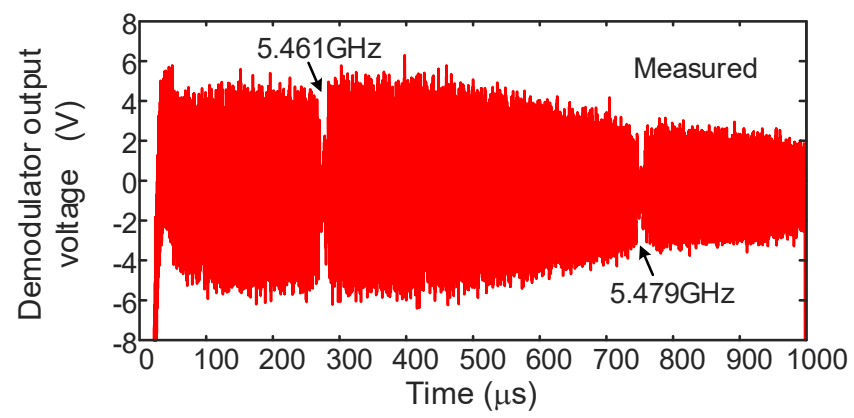

(a)

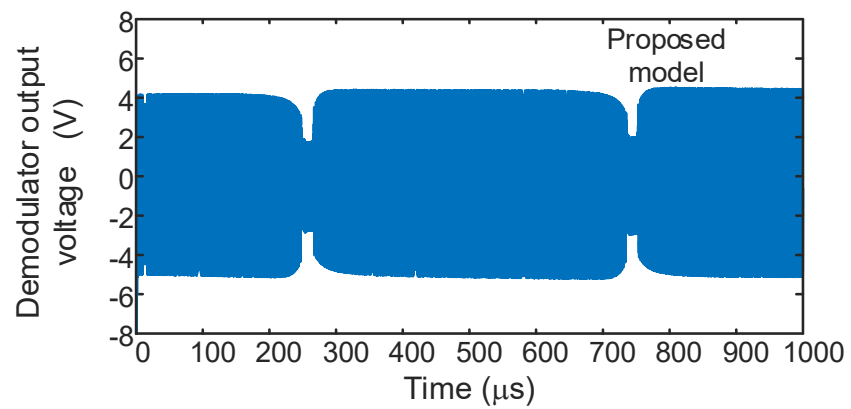

(b)

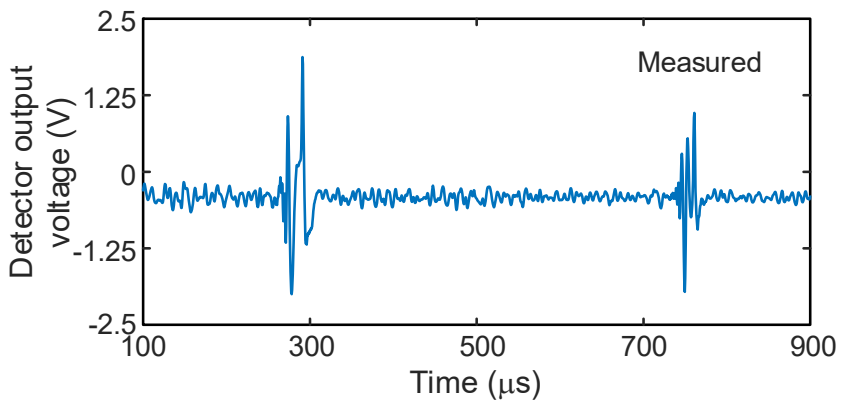

(c)

Fig. 6. Detection of two input signals for input power below the one required to reach any locked states. (a)-(b) Comparison between measurement and analysis results. (c) Output of the envelope detector.

\section{CONCLUSIONS}

A two-scale envelope-domain formulation for injected chirped oscillators, usable under a low chirp frequency and relatively high beat frequencies, has been presented. The formulation accurately predicts the magnitude and frequency variations of the oscillation signal during the chirp period. The behavior under several input signals has also been successfully predicted, and the capability to detect the input frequencies from the dynamics of the beat frequency has been demonstrated.

\section{ACKNOWLEDGMENT}

This work was supported by the Spanish Ministry of Economy and Competitiveness and the European Regional Development Fund (ERDF/FEDER) under research projects TEC2014-60283-C3-1-R and TEC2017-88242-C3-1-R.

\section{REFERENCES}

[1] F. K. Wang et al., "An injection-locked detector for concurrent spectrum and vital sign sensing," IEEE MTT-S Int. Microwave Symp. Dig., Anaheim, CA, 2010, pp. 768-771.

[2] C. J. Li, F. K. Wang, T. S. Horng and K. C. Peng, "A Novel RF Sensing Circuit Using Injection Locking and Frequency Demodulation for Cognitive Radio Applications," IEEE Trans. Microw. Theory Techn., vol. 57, no. 12, pp. 3143-3152, Dec., 2009.

[3] D. T. Lin, H. Chae, L. Li and M. P. Flynn, "A Low-Power Adaptive Receiver Utilizing Discrete-Time Spectrum-Sensing," IEEE Trans. Microw. Theory Techn., vol. 61, no. 3, pp. 1338-1346, March 2013.

[4] Tae Won Ban and Wan Choi, "Opportunistic spectrum sensing in cognitive radio systems," 2011 IEEE MTT-S International Microwave Workshop Series on Intelligent Radio for Future Personal Terminals, Daejeon, 2011, pp. 1-2.

[5] A. Suárez, F. Ramírez, S. Sancho, "Stability and Noise Analysis of Coupled-Oscillator Systems," IEEE Trans. Microw. Theory Techn., vol. 59, no. 4, pp. 1032-1046, 2011.

[6] A. Suárez, F. Ramírez, "Analysis of stabilization circuits for phasenoise reduction in microwave oscillators", IEEE Trans. Microw. Theory Techn., vol. 53, no. 9, pp. 2743-2751, Sept., 2005.

[7] E. Ngoya and R. Larcheveque, "Envelop transient analysis: a new method for the transient and steady state analysis of microwave communication circuits and systems," IEEE MTT-S Int. Microwave Symp. Dig., San Francisco, CA, USA, 1996, vol.3, pp. 1365-1368.

[8] J. C. Pedro and N. B. Carvalho, "Simulation of RF circuits driven by modulated signals without bandwidth constraints," IEEE MTT-S Int. Microwave Symp. Dig., Seattle, WA, USA, 2002, pp. 2173-2176 vol.3.

[9] E. de Cos, A. Suárez and S. Sancho, "Envelope transient analysis of self-oscillating mixers," IEEE Trans. Microw. Theory Techn., vol. 52, pp. 1090-1100, 2004.

[10] S. Sancho, M. Pontón, A. Suárez, "Effects of Noisy and Modulated Interferers on the Free-Running Oscillator Spectrum," IEEE Trans. Microw. Theory Techn., vol. 66, 2018. doi: 10.1109/TMTT.2017.2786700.

[11] S. Sancho, F. Ramírez, A. Suárez, "Stochastic Analysis of Cycle Slips in Injection-Locked Oscillators and Analog Frequency Dividers," IEEE Trans. Microw. Theory Techn., vol. 62, pp. 3318-3332, 2014. 